\title{
Eddy turbulence parameters inferred from radar observations at Jicamarca
}

\author{
M. N. Vlasov ${ }^{1}$, M. C. Kelley ${ }^{1}$, and D. L. Hysell ${ }^{2}$ \\ ${ }^{1}$ School of Electrical and Computer Engineering, Cornell University, 320 Rhodes Hall, Ithaca, New York, 14853, USA \\ ${ }^{2}$ Department of Earth and Atmospheric Science, Cornell University, 2108 Snee Hall, Ithaca, New York, 14853, USA
}

Received: 29 September 2006 - Revised: 13 February 2007 - Accepted: 14 February 2007 - Published: 8 March 2007

\begin{abstract}
Significant electron density striations, neutral temperatures $27 \mathrm{~K}$ above nominal, and intense wind shear were observed in the E-region ionosphere over the Jicamarca Radio Observatory during an unusual event on 26 July 2005 (Hysell et al., 2007). In this paper, these results are used to estimate eddy turbulence parameters and their effects. Models for the thermal balance in the mesosphere/lower thermosphere and the charged particle density in the E region are developed here. The thermal balance model includes eddy conduction and viscous dissipation of turbulent energy as well as cooling by infrared radiation. The production and recombination of ions and electrons in the E region, together with the production and transport of nitric oxide, are included in the plasma density model. Good agreement between the model results and the experimental data is obtained for an eddy diffusion coefficient of about $1 \times 10^{3} \mathrm{~m}^{2} / \mathrm{s}$ at its peak, which occurs at an altitude of $107 \mathrm{~km}$. This eddy turbulence results in a local maximum of the temperature in the upper mesosphere/lower thermosphere and could correspond either to an unusually high mesopause or to a double mesosphere. Although complicated by plasma dynamic effects and ongoing controversy, our interpretation of Farley-Buneman wave phase velocity (Hysell et al., 2007) is consistent with a low Brunt-Väisälä frequency in the region of interest. Nitric oxide transport due to eddy diffusion from the lower thermosphere to the mesosphere causes electron density changes in the $\mathrm{E}$ region whereas $\mathrm{NO}$ density modulation due to irregularities in the eddy diffusion coefficient creates variability in the electron density.
\end{abstract}

Keywords. Ionosphere (Equatorial ionosphere; Midlatitude ionosphere; Plasma waves and instabilities)

Correspondence to: M. C. Kelley

(mikek@ece.cornell.edu)

\section{Introduction}

Turbulence plays an important role in the lower thermosphere and mesosphere (Banks and Kockarts, 1973). Eddy turbulence can cool or heat a gas due to eddy conduction and also can heat a gas through viscous dissipation of turbulent kinetic energy. Eddy diffusion provides mixing of the main atmospheric components, which leads to partial ratios independent of altitude. This diffusion transports minor constituents. The latter effect can influence the thermospheric density and temperature at high altitudes. An increase in eddy diffusion decreases the density of atomic oxygen due to transport to regions of high recombination. This decrease then propagates to high altitudes where atomic oxygen is the main component. The eddy transport of NO influences the thermal balance in the thermosphere because of cooling by NO infrared radiation and the plasma density by changing the $[\mathrm{O}] /\left[\mathrm{O}_{2}\right]$ ratio.

Eddy diffusion can exist if the scales of eddy mixing are much larger than the mean free path and much smaller than the atmospheric scale height. As shown in Fig. 1, the range of eddy scales corresponding to this condition is very narrow at $120 \mathrm{~km}$ but expands with decreasing altitude. Thus, according to this criterion, eddy turbulence can develop at altitudes below $120 \mathrm{~km}$ but is unlikely above this altitude. This rough estimate of the upper boundary of the eddy turbulence is in reasonable agreement with measurements of the turbopause altitude, where molecular diffusion starts to dominate eddy mixing.

Radar scatter from atmospheric turbulence has provided many insights into the dynamics of the mesosphere, stratosphere, and troposphere. Above the mesosphere, turbulent scatter has been less useful for two reasons. First, in the lower thermosphere, turbulence is less active according to the criterion discussed above and the atmosphere is in transition from a fully mixed medium to one in which the constituents separate according to their mass. Second, the index

Published by Copernicus GmbH on behalf of the European Geosciences Union. 


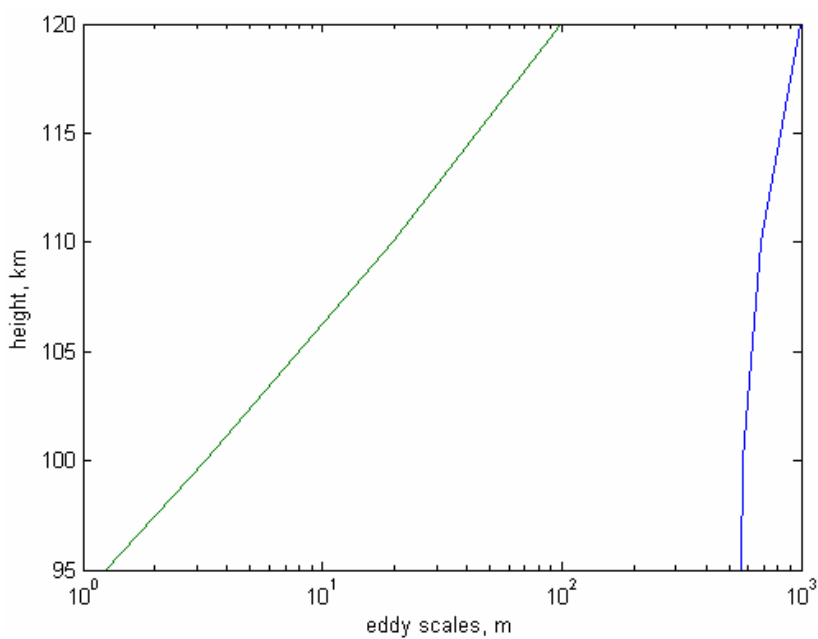

Fig. 1. Height distribution of the eddy scale maximum and minimum for eddy diffusion.

of refraction becomes dominated by the electron density in the collocated ionosphere such that plasma instabilities may mask the underlying turbulence of the medium. This is particularly true at the magnetic equator where one of the largest VHF radar systems operates: the Jicamarca $50 \mathrm{MHz}$ radar. Here, the scatter between 95 and $120 \mathrm{~km}$ is very much dominated by plasma instabilities in the equatorial electrojet. However, the daytime equatorial ionosphere has been studied for decades, particularly with respect to these plasma instabilities, and theoretical bases exist to interpret these data in terms of the underlying winds and temperatures of the neutral atmosphere. We take advantage of these new insights here to study a very unusual data set that suggests a high level of turbulence between 100 and $110 \mathrm{~km}$, suggesting either a very high mesopause or a double mesopause.

In the past few years new experimental approaches to studying the daytime equatorial electrojet have been developed that overcome many of the limitations imposed by plasma processes (see companion paper Hysell et al., 2007). These limitations include Faraday rotation measurements using the plasma waves as scatterers on an oblique path (Hysell and Chau, 2002; Shume et al., 2005b); an improved ability to image plasma structures in the unstable region with interferometric methods (Hysell and Chau, 2006); an inversion technique to deduce neutral winds from the Doppler shift of oblique plasma waves (Shume et al., 2005a); identification of the Doppler shift of " $150 \mathrm{~km}$ echoes" with $\boldsymbol{E} \times \boldsymbol{B}$ drifts due to the zonal electric field (Kudeki and Fawcett, 1993); and establishment of a new AMISR prototype radar at $430 \mathrm{MHz}$ at Jicamarca (Cuevas et al., 2007 ${ }^{1}$ ). Hysell et al. (2007) re-

\footnotetext{
${ }^{1}$ Cuevas, R., Kelley, M. C., Chau, J. L., and Heinselman, C.: The seven-panel advanced modular scatter radar at the Jicamarca Radio Observatory: System overview and initial results, J. Geophys. Res., submitted, 2007.
}

ported the first combined results using these systems. Some of these results were surprising, including the topic discussed in more detail in this paper.

The primary result of interest here is reproduced in Fig. 2. Instead of a smooth E-region plasma density profile, the Faraday rotation data indicate a very patchy $\mathrm{E}$ region even in the face of daytime production, which should seemingly destroy any depleted regions. The data indicate layers separated by $3-5 \mathrm{~km}$ in altitude and $90 \mathrm{~min}$. in time, which propagated slowly downward with a period of about $90 \mathrm{~min}$. Several observations discussed by Hysell et al. (2007) have led to the idea explored here that the $100-110 \mathrm{~km}$ region was subject to a neutral atmosphere instability which, we argue, can yield neutral atmospheric heating and plasma layering.

1. Measurements of the ion acoustic speed at two different radar frequencies indicate a temperature of 210 degrees at $102 \mathrm{~km}$, higher than the MSIS-E-90 model predicts (Hedin, 1991).

2. The inversion method for neutral winds shows a large scale, long-period wind field with an associated velocity shear of $30 \mathrm{~m} / \mathrm{s}$ per $\mathrm{km}$.

3. The downward phase progression in the plasma density and its periodicity indicate upward energy propagation in a gravity wave mode.

The goal of this paper is to describe the observed temperature enhancement in the upper mesosphere and the disturbance of electron density in the $\mathrm{E}$ region using a one-dimensional model with eddy heating and conductivity, chemical and UV heating, and radiative cooling. Finally, the parameters of the eddy diffusion coefficient required to produce the observed changes are inferred.

\section{Instability of the neutral atmosphere}

Three neutral instability mechanisms have been proposed for the mesosphere. Convective instability occurs when the square of the Brunt-Väisälä frequency, $\omega_{B}^{2}$, is negative, requiring a temperature gradient more negative than $-9.5 \mathrm{de}$ grees $/ \mathrm{km}$. Observation 1 above suggests that a negative temperature gradient existed but may not correspond to convective instability.

The second mechanism is the Kelvin-Helmholtz instability (KHI), which occurs when the Richardson number is less than $1 / 4$. This parameter is given by expressions

$$
R_{i}=\frac{\omega_{B}^{2}}{(d U / d z)^{2}}
$$

and

$$
\omega_{B}^{2}=\frac{g}{T}\left(\frac{d T}{d z}+\frac{g}{C_{p}}\right),
$$




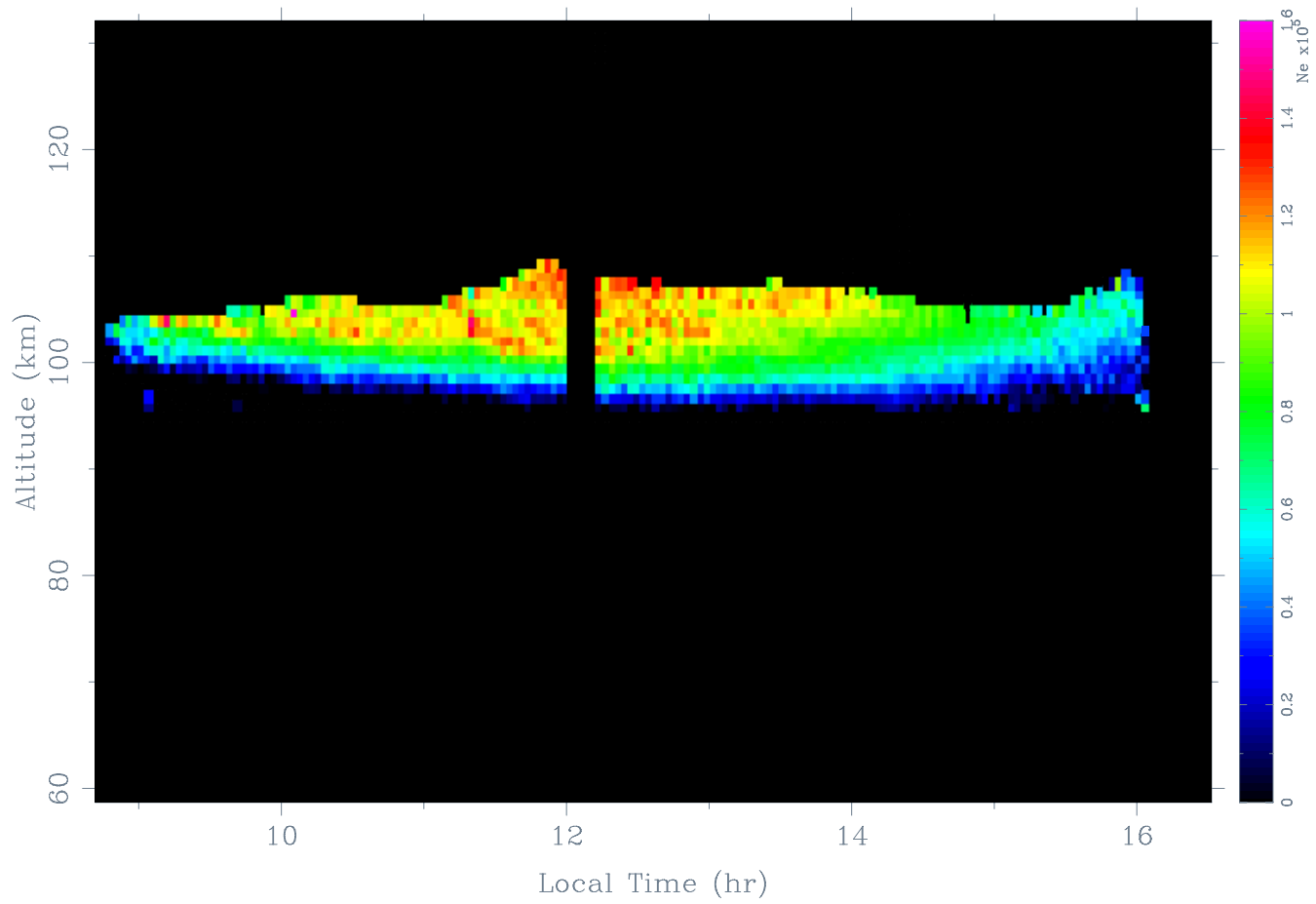

Fig. 2. Electron density measured by radar (color plot).

where $U$ is the horizontal wind velocity, $g$ is the gravitational acceleration, $C_{p}$ is the specific heat at constant pressure, and $T$ is the temperature. The term in the denominator of Eq. (1) is the square of the wind shear. For the temperature gradient we conservatively use the MSIS-E-90 model, which yields $\omega_{B}^{2}=4.5 \times 10^{-4} \mathrm{~s}^{-2}$ at $95 \mathrm{~km}$. Instability occurs for wind shears greater than $45 \mathrm{~m} / \mathrm{s}$ per $\mathrm{km}$. The data reported by Hysell et al. (2007) for the case considered here show that the shear is $30 \mathrm{~m} / \mathrm{s}$ per $\mathrm{km}$ between 95 and $102 \mathrm{~km}$. This shear value is not out of the ordinary. Larsen (2002) reported that the average shear between 100 and $110 \mathrm{~km}$ is in the range of $20-30 \mathrm{~m} / \mathrm{s}$ per $\mathrm{km}$. Thus, the regions of low convective stability are close to being KHI unstable. It is easy to see in this case that the background wind shear, which is roughly semi-diurnal in character, is dynamically unstable to the parameter variations due to passing gravity waves and was in this high shear condition for many hours.

A third mechanism is the turning point instability first discussed in the atmospheric boundary layer (Brown, 1970, 1980) and first introduced to the mesospheric domain by Larsen et al. (2004). In this case a region of low stability in the presence of a shear with an inflection point will create a long-period (e.g., 60-90 min.) overturning of the layer with vertical scales in the mesosphere of $2-3 \mathrm{~km}$. This process, in conjunction with the KHI, may be responsible for the turbulence we need to explain the data.

\section{Analysis of the effects of turbulence}

The turbopause is a statistical concept with an average altitude thought to be in the range around $100-110 \mathrm{~km}$. However, near the statistical turbopause, at any given time there can be regions of high eddy diffusion above regions with no mixing. This is demonstrated by the appearance of long-lived meteor trails, as presented by Kelley et al. (2003). This variability may be associated with the phase of tides or gravity waves propagating through the region, which seems to be the case for the event discussed here.

We argue below that both the temperature increase indicated by the ion acoustic speed and the structure in the electron density in this event may well be related to neutral gas instability as outlined above. It is well known that a temperature enhancement can be caused by dissipation of turbulent energy (Gordiets et al., 1982). The eddy diffusion associated with the turbulence can in turn strongly influence the nitric oxide profile due to the very long chemical lifetime of the species. In turn, the striations can result from localized turbulent transport of this minor constituent, since nitric oxide influences ion composition and electron density in the E region. 


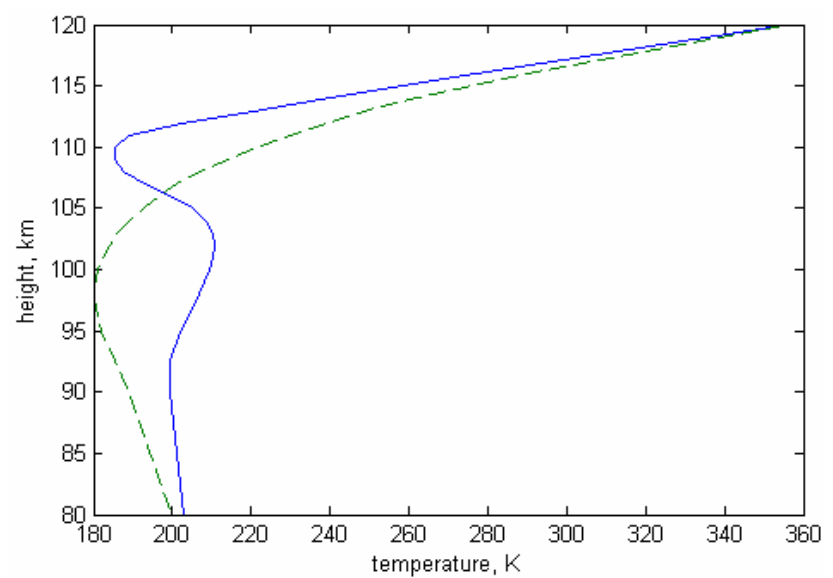

Fig. 3. Height profiles of the neutral temperature: the background profile given by the MSIS-E-90 model (dashed curve) and the profile calculated by the model (solid curve).

3.1 Consistency of temperature and turbulence characteristics indicated by radar data with the theory of turbulent heating

Let us first consider a connection between the temperature enhancement, the turbulent energy dissipation, and the associated eddy diffusion. The eddy diffusion coefficient $K_{\text {ed }}$ may be couched as a physical analog of the molecular diffusion coefficient (Hocking, 1987). The latter is given by the formula

$D=\kappa T / m v$,

where $\kappa$ is the Boltzmann constant, $T$ is the temperature, $m$ is the atomic mass, and $v$ is the collision frequency. Using the turbulent energy per mass dissipated during the vertical relaxation time of an air parcel,

$E=\varepsilon / \omega_{B}$,

instead of the thermal energy per mass, and buoyancy frequency $\omega_{B}$ instead of the collision frequency, it is possible to derive a formula for the eddy diffusion coefficient,

$K_{e d}=C \varepsilon / \omega_{B}^{2}$,

where $\varepsilon$ is the turbulent energy dissipation rate and $C$ is a constant of order unity, generally taken to be between 0.2 and 1.

At mesospheric heights $g / C_{p}=9.5 \mathrm{~K} / \mathrm{km}$ and, as mentioned above, convective instability occurs for temperature gradients more negative in absolute value than this number. The nominal height profile of the temperature given by the MSIS-E-90 model is shown in Fig. 3, which has a minimum (the mesopause) at altitudes at or below $100 \mathrm{~km}$, a sharp increase in the temperature above the mesopause, and a small negative gradient below the mesopause. This profile has a stable mesosphere and lower thermosphere $\left(\omega_{B}^{2}>0\right)$. The observations show a temperature peak of $210 \mathrm{~K}$ at the altitude of $102 \mathrm{~km}$. According to Vlasov and Korobeynikova (1991), a temperature peak at any height in the upper mesosphere can be produced by dissipation of the turbulent energy if the eddy diffusion coefficient is larger than $6 \times 10^{2} \mathrm{~m}^{2} \mathrm{~s}^{-1}$ at the $K_{e d}$ peak. The altitude of the $K_{e d}$ peak should be higher than the altitude of the temperature peak. This means that, in our case, the altitude of the $K_{e d}$ peak must be near $110 \mathrm{~km}$, which in turn means that the mesopause was higher than usual or that a double mesopause occurred on this day, which is a fairly common occurrence. In any case the observations suggest a strong disturbance of the mesosphere.

With such a high temperature there is most likely a steep gradient (lapse rate) in the temperature at altitudes above $102 \mathrm{~km}$, and the buoyancy frequency squared may be very small. This implies an increase in the eddy diffusion coefficient according to Eq. (5). The heating rate due to the dissipation of turbulent energy is given by the relation (Gordiets et al., 1982):

$Q_{e d H}=\varepsilon \rho=K_{e d} \rho \omega_{B}^{2} /\left(\alpha_{i} R_{i}\right)$,

where $\rho$ is the neutral density and $\alpha_{i}=0.3$. The divergence of the zonal neutral wind inferred from the measurements of the Doppler shifts during the event was as high as $30 \mathrm{~m} / \mathrm{s}$ per $\mathrm{km}$ over a time period indicative of a diurnal tide (Hysell et al., 2007). For such long time scales we consider the time-independent equation of the thermal balance in the mesosphere, which can be written as

$$
\begin{aligned}
\frac{\partial}{\partial z}\left[K_{e d} C_{p} \rho\left(\frac{\partial T}{\partial z}+\frac{g}{C_{p}}\right)\right]= & Q_{c h}+K_{e d} \rho \frac{g}{T R_{i} \alpha_{i}} \\
& \left(\frac{\partial T}{\partial z}+\frac{g}{C_{p}}\right)-L_{I R},
\end{aligned}
$$

where $Q_{c h}$ is heating due to chemical reactions and $L_{I R}$ is cooling by infrared radiation. Infrared radiation in $15 \mu$ bands of $\mathrm{CO}_{2}$ is the main sink of energy in the mesosphere. $K_{e d}$ is assumed to be equal to the eddy heat conductivity. We use the approximation of the eddy coefficient given by Shimazaki (1971):

$$
\begin{aligned}
K_{e d}(z)= & K_{e d}^{0} \exp \left[S_{1}\left(z-z_{m}\right)\right]+\left(K_{e d}^{m}-K_{e d}^{0}\right) \\
& \exp \left[-S_{2}\left(z-z_{m}\right)^{2}\right] \quad z \leq z_{m}, \\
K_{e d}(z)= & K_{e d}^{m} \exp \left[-S_{3}\left(z-z_{m}\right)^{2}\right] \quad z>z_{m},
\end{aligned}
$$

where $K_{e d}^{m}$ and $K_{e d}^{0}$ are the eddy diffusion coefficients at the altitude of the peak, $z_{m}$, and at the low boundary, respectively, $S_{1}, S_{2}$, and $S_{3}$ are free parameters. The numerical solution of Eq. (7) facilitates estimating the eddy diffusion coefficient corresponding to the observed temperature peak. The $K_{e d}$ height profile is shown in Fig. 4 and the temperature height profile calculated by the model is shown in Fig. 3. The $K_{e d}^{m}$ maximum value is $1 \times 10^{3} \mathrm{~m}^{2} / \mathrm{s}$. This maximum value is 
comparable to the value deduced from the meteor trail expansion near $95 \mathrm{~km}$ (Kelley et al., 2003). The gradient of the eddy diffusion coefficient below the peak is determined by parameters $S_{1}$ and $S_{2}$. The normal values of these parameters, $S_{1}=0.07 \mathrm{~km}^{-1}$ and $S_{2}=0.03 \mathrm{~km}^{-2}$, are used. The $S_{3}$ value, found to be $0.05 \mathrm{~km}^{-2}$, influences the peak of the temperature in the mesosphere. According to our calculations, eddy turbulence with these parameters can provide the observed enhancement of the temperature in the mesosphere.

\subsection{Relationship between turbulent transport and plasma density structures}

We now turn to considering the impact of turbulence on the nitric oxide and plasma density profiles. The principal source of nitric oxide in the lower thermosphere is the chemical reaction between excited atomic nitrogen and molecular oxygen,

$\mathrm{N}\left({ }^{2} \mathrm{D}\right)+\mathrm{O}_{2} \rightarrow \mathrm{NO}+\mathrm{O}$.

There is another loss mechanism for excited atomic nitrogen, namely, the deactivation reaction with atomic oxygen given by

$\mathrm{N}\left({ }^{2} \mathrm{D}\right)+\mathrm{O} \rightarrow \mathrm{N}\left({ }^{+} \mathrm{S}\right)+\mathrm{O}$.

Both reactions are fast, and the $\mathrm{N}\left({ }^{2} \mathrm{D}\right)$ density is thus very sensitive to the $[\mathrm{O}] /\left[\mathrm{O}_{2}\right]$ ratio.

Note that Reaction (10) is negligible in the balance of atomic oxygen. It is well known that an increase in the eddy diffusion induces a decrease in the atomic oxygen density due to transport of atomic oxygen to low altitudes where three-body recombination of $\mathrm{O}$ atoms is very effective. The eddy diffusion coefficient estimated above can induce a significant decrease in $[\mathrm{O}]$ and thus an increase in the NO production rate due to Reaction (10). At the same time this eddy diffusion provides a transport mechanism for nitric oxide flux from the lower thermosphere to the mesosphere.

We now consider the photochemistry of the E region in detail. Ionization of molecular oxygen by solar radiation is the main source of charged particles in this region. The ionization rate of $\mathrm{N}_{2}$ does not exceed $10 \%$ of the $\mathrm{O}_{2}$ ionization rate. The $\mathrm{N}_{2}^{+}$reactions with atomic and molecular oxygen are very fast, and these ions are transformed into $\mathrm{O}_{2}^{+}$and $\mathrm{NO}^{+}$ions before their recombination. Thus, the small ionization rate of $\mathrm{N}_{2}$ and the effective loss of $\mathrm{N}_{2}^{+}$make the $\mathrm{N}_{2}^{+}$density negligible. The resulting $\mathrm{O}_{2}^{+}$and $\mathrm{NO}^{+}$ions dominate the ion composition in the E region. The main source of $\mathrm{NO}^{+}$is the reaction

$\mathrm{O}_{2}^{+}+\mathrm{NO} \rightarrow \mathrm{NO}^{+}+\mathrm{O}$.

The $\mathrm{O}_{2}^{+}$loss due to this reaction can be more important than dissociative recombination if the NO density is higher than $3 \times 10^{7} \mathrm{~cm}^{-3}$. Taking into account the processes discussed

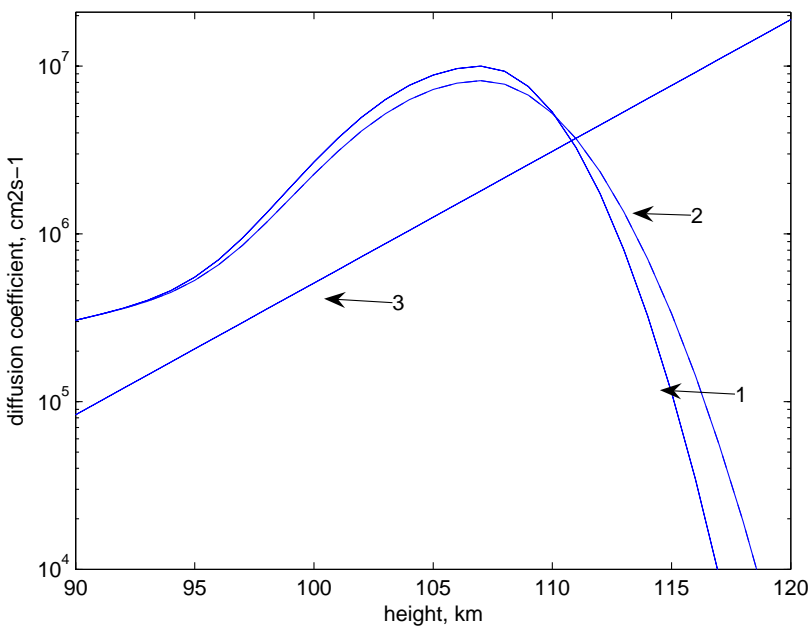

Fig. 4. Height profiles of eddy diffusion coefficients corresponding to the observed temperature enhancement (curve 1), the observed disturbances of electron density (curve 2), and the molecular diffusion coefficient (curve 3).

above, the formulas for the ion and electron densities in the E region are given by

$\left[\mathrm{O}_{2}^{+}\right]=\frac{q_{\mathrm{O}_{2}^{+}}+k q_{\mathrm{N}_{2}^{+}}}{\alpha_{\mathrm{O}_{2}^{+}}^{8} N_{e}+\gamma_{1}[\mathrm{NO}]}$,
$\left[\mathrm{NO}^{+}\right]=\frac{(1-\kappa) q_{\mathrm{N}_{2}^{+}}+\left[\mathrm{O}_{2}^{+}\right] \gamma_{1}[\mathrm{NO}]}{\alpha_{\mathrm{NO}^{+}}^{*} N_{e}}$,

$N_{e}=\left[\mathrm{O}_{2}^{+}\right]+\left[\mathrm{NO}^{+}\right]$,

where $k$ is a portion of the $\mathrm{O}_{2}^{+}$production from the $\mathrm{N}_{2}$ ionization rate, $q_{\mathrm{O}_{2}^{+}}$and $q_{\mathrm{N}_{2}^{+}}$are the ionization rates of $\mathrm{O}_{2}$ and $\mathrm{N}_{2}$, respectively, $\gamma_{1}$ is the rate coefficient of Reaction (10), and $\alpha_{\mathrm{O}_{2}^{+}}^{*}$ and $\alpha_{\mathrm{NO}^{+}}^{*}$ are dissociative recombination coefficients for the $\mathrm{O}_{2}^{+}$and $\mathrm{NO}^{+}$ions. Using these formulas, we obtain an equation for the electron density:

$$
\begin{gathered}
\alpha_{\mathrm{O}_{2}^{+}}^{*} \alpha_{\mathrm{NO}^{+}}^{*} N_{e}^{3}+\alpha_{\mathrm{NO}^{+}}^{*} \gamma_{1}[\mathrm{NO}] N_{e}^{2}-\left[\left(q_{\mathrm{O}_{2}^{+}}+\kappa q_{\mathrm{N}_{2}^{+}}\right) \alpha_{\mathrm{NO}^{+}}^{*}\right. \\
\left.+(1-\kappa) q_{\mathrm{N}_{2}^{+}} \alpha_{\mathrm{O}_{2}^{+}}^{*}\right] N_{e}-\left(q_{\mathrm{O}_{2}^{+}}+q_{\mathrm{N}_{2}^{+}}\right) \gamma_{1}[\mathrm{NO}]=0 .
\end{gathered}
$$

For this day there is good agreement between the measured mean electron density (Hysell et al., 2007) and the density calculated by the IRI model (Bilitza, 2001). This agreement supports using the ion composition and electron density given by the IRI model to estimate ionization rates. These ionization rates can be used in Eq. (16) because the observed striations and temperature enhancement do not have any apparent relationship to changes in solar radiation. The electron densities calculated by Eq. (16), using different densities of NO and recombination coefficients corresponding to 
the measured temperature, indicate that an increase by a factor of 1.4 in the NO density induces an electron density decrease of $20 \%$. This electron density fluctuation is in agreement with the observed striations shown in Fig. 2. Our model shows that the enhancement of NO can result from eddy diffusion with $K_{e d}^{m}=8.7 \times 10^{2} \mathrm{~m}^{2} / \mathrm{s}$. This enhancement can be modulated by fluctuations of the eddy turbulence associated with gravity wave modulation of the Richardson number; this modulation induces the electron density fluctuations. Eddy diffusion coefficient modulation has been observed by Zimmerman and Murphy (1977) and by Kelley et al. (2003).

According to Vlasov and Davydov (1991), the interaction between eddy and molecular diffusion, together with photochemical processes, can induce a splitting of the atomic oxygen height profile. This splitting is reflected in the NO height profile due to Reaction (10). Note that the striations decrease with decreasing altitude and that the NO density decreases sharply with decreasing altitude as well. Also, the strongest striations are observed around noon when the NO density is at a maximum.

There are small differences between the eddy diffusion coefficient inferred from the temperature enhancement and the coefficient inferred from the NO enhancement (see Fig. 4). The $K_{e d}^{m}$ value corresponding to the temperature enhancement is larger by a factor of 1.2 than the $K_{e d}^{m}$ value corresponding to the NO enhancement. Also, the gradient of the eddy diffusion coefficient above the peak for the temperature enhancement is steeper than the gradient for the NO enhancement. The main cause of these differences is an uncertainty in the model for the thermal balance since there are feedbacks between the eddy diffusion and the radiative cooling. It is very difficult to obtain a self-consistent solution for this problem. There are also significant uncertainties for both the $R_{i}$ value and the rate coefficient for the reaction of vibrationally excited $\mathrm{CO}_{2}$ with atomic oxygen. The impact of this rate coefficient on radiative cooling is very complicated. We think that the difference between the calculated values is in reasonable agreement with the accuracy of the thermal balance modeling.

A very unusual temperature profile measured by the Starfire sodium resonance lidar was presented by Kelley et al. (2003). The profile has the temperature peak at $89 \mathrm{~km}$ and a mesopause at $100 \mathrm{~km}$. The temperature enhancement in the peak was $36 \mathrm{~K}$, and several regions of convective and dynamic instability were found. According to our model, a $K_{e d}^{m}$ value larger than $1 \times 10^{3} \mathrm{~m}^{2} / \mathrm{s}$ may be needed to provide the temperature enhancement observed at altitudes below $90 \mathrm{~km}$. This level of eddy diffusion is in agreement with the observed expansion of long-lived Leonid meteor trails seen during that same event.

\section{Conclusions}

New instrumentation and data analysis techniques at the Jicamarca Radio Observatory were used to study the equatorial ionosphere and lower thermosphere during several days in 2005. On one of these days, a remarkable variability in the plasma density profiles was observed in the height range from 100-108 km. In addition, measurements of the acoustic speed of plasma waves indicated a temperature that is nearly $27 \mathrm{~K}$ above nominal. Furthermore, wind variations deduced from multiple beam experiments indicate a long-lasting intense shear. Taken together, these data suggest regions of long-lasting turbulence organized by the combination of a tidal mode and a gravity wave with a period of $90 \mathrm{~min}$. Modeling the observed mesospheric temperature enhancement requires an eddy diffusion coefficient of $9.7 \times 10^{2} \mathrm{~m}^{2} / \mathrm{s}$ with an altitude peak of $107 \mathrm{~km}$. The corresponding turbulent heating and cooling creates a temperature peak in the upper mesosphere and a mesopause at $109 \mathrm{~km}$ altitude. This is either a change in the mesopause or a double mesopause.

In turn, the NO transport due to eddy diffusion from the lower thermosphere to the mesosphere is the likely source of change in the electron density in the E region. A small difference exists between the height distribution of the eddy diffusion coefficient corresponding to both the observed temperature and the NO density. This difference is still within the accuracy limits of the two models used here. The passage of gravity waves may create irregularities in the eddy diffusion height distribution with scales of a few kilometers. This distribution agrees with observations of vertical structure in the plasma density. We also associate these features with eddy transport of atomic oxygen and a corresponding variability in NO density which, in turn, leads to the observed plasma ion recombination and striations.

A similar evolution of a shear flow region was reported using radar and balloon data from the upper troposphere (Kelley et al., 2005). In this case, shear was disrupted over many hours by localized instabilities due to gravity waves. Patches of turbulence within the sheared flow with small Brunt-Väisälä frequencies (i.e., at the threshold for convective instability) were created. Such a process may be occurring in the current data set.

Acknowledgements. Work at Cornell University was supported by the Air Force Office of Scientific Research under grant FA955005-0160 and by the Atmospheric Science Section of the National Science Foundation under grant ATM-0000196.

Topical Editor M. Pinnock thanks one referee for her/his help in evaluating this paper.

\section{References}

Banks, P. M. and Kockarts, G.: Aeronomy, Part A, Academic Press, San Diego, Calif., 1973.

Bilitza, D.: International reference ionosphere, Radio Sci., 36, 261275, 2001. 
Brown, R. A.: A secondary flow model for the preliminary boundary layers, J. Atmos. Sci., 27, 742-757, 1970.

Brown, R. A.: Longitudinal instabilities and secondary flows in the planetary boundary layer: A review, Rev. Geophys., 18, 683697, 1980.

Gordiets, B. F., Kulikov, Yu. N., Markov, M. N., and Markov, M. Ya.: Numerical modeling of the thermospheric heat budget, J. Geophys. Res., 87, 4504-4514, 1982.

Hedin, A. E.: Extension of the MSIS thermospheric model into the middle and lower atmosphere, J. Geophys. Res., 96, 1159-1172, 1991.

Hocking, W.: Turbulence in the region 80-120 km, Adv. Space Res., 7, 171-181, 1987.

Hysell, D. L. and Chau, J. L.: Imaging radar observations and nonlocal theory of large-scale waves in the equatorial electrojet, Ann. Geophys., 20, 1167-1179, 2002, http://www.ann-geophys.net/20/1167/2002/.

Hysell, D. L. and Chau, J. L.: Optimal aperture synthesis radar imaging, Radio Sci., 41, RS2003, doi:10.1029/2005RS003383, 2006.

Hysell, D. L., Drexler, J., Shume, E. B., Chau, J. L., Scipion, D. E., Vlasov, M., Cuevas, R., and Heinselman, C.: Combined radar observations of equatorial electrojet irregularities at Jicamarca, Ann. Geophys., 25, 457-473, 2007, http://www.ann-geophys.net/25/457/2007/.

Kelley, M. C., Kruschwitz, C. A., Gardner, C. S., Drummond, J. D., and Kane, T. J.: Mesospheric turbulence measurements from persistent Leonid meteor train observations, J. Geophys. Res., 108, 8454, doi:10.1029/2002JD002392, 2003.

Kelley, M. C., Chen, C. Y., Beland, R. R., Woodman, R., Chau, J. L., and Werne, J.: Persistence of a Kelvin-Helmholtz instability complex in the upper troposphere, J. Geophys. Res., 110, D14106, doi:10.1029/2004JD005345, 2005.
Kudeki, E. and Fawcett, C. D.: High resolution observations of $150 \mathrm{~km}$ echoes at Jicamarca, Geophys. Res. Lett., 20, 19871990, 1993.

Larsen, M. F.: Winds and shears in the mesosphere and lower thermosphere: Results from four decades of chemical release wind measurements, J. Geophys. Res., 107, 1215, doi:10.1029/2001JA000218, 2002.

Larsen, M. F., Liu, A. Z., Gadner, C. S., Kelley, M. C., Collins, S., Friedman, J., and Hecht, J. H.: Observations of overturning in the upper mesosphere and lower thermosphere, J. Geophys. Res., 109, D02S04, 10.1029/2002JD003 067, 2004.

Shimazaki, T.: Effective eddy diffusion coefficient and atmospheric composition in the lower thermosphere, J. Atmos. Terr. Phys., 33, 1383-1401, 1971.

Shume, E. B., Hysell, D. L., and Chau, J. L.: Zonal wind velocity profiles in the equatorial electrojet derived from phase velocity of type II radar echoes, J. Geophys. Res., 110, A12308, doi:10.1029/2005JA011210, 2005a.

Shume, E. B., Hysell, D. L., and Chau, J. L.: Electron density profiles in the equatorial E-region ionosphere derived from a bistatic coherent scatter radar experiment in Peru, Geophys. Res. Lett., 32, L01107, doi:10.1029/2004GL021715, 2005b.

Vlasov, M. N. and Davydov, V. E.: Splitting of atomic oxygen height profiles by eddy diffusion, J. Atmos. Terr. Phys., 55, 1361-1364, 1991.

Vlasov, M. N. and Korobeynikova, T. V.: Influence of turbulence, infrared radiation and mass-averaged transport on the height distribution of the temperature in middle and high latitude thermosphere, Cosmic Res., 29, 544-550, 1991.

Zimmerman, S. P. and Murphy, E. A.: Dynamical and chemical coupling, D. Reidel, Dordrecht, Holland, 1977. 